\title{
The Current Advance of Clinical Drug for Treatment Alzheimer's Disease and the Research of New Drug
}

\author{
DING Sheng kai ${ }^{1}$ and SHANG Ya-zhen ${ }^{2 *}$ \\ ${ }^{1}$ Hebei Province Key Laboratory office of Traditional Chinese Medicine Against Dementia, China \\ ${ }^{2}$ Hebei Province Key Laboratory of Traditional Chinese Medicine Research and Development, China
}

*Corresponding author: Shang Yazhen, Institute of Traditional Chinese

Medicine, Chengde Medical College, Hebei, Chengde 067000, China.

Received Date: April 11, 2020

Published Date: September 28, 2020

\begin{abstract}
Alzheimer's disease (AD) is a typical degenerative disease of the central nervous system. Along with the continuous deepening of the aging of the population, the prevalence rate of $\mathrm{AD}$ is also increasing year by year. According to the national epidemiological survey of mental illness, the prevalence rate of Alzheimer's disease among people aged 65 and over in China is 5.56\%. It is estimated that the number of AD patients worldwide will increase to 152 million by 2050. So far, only 5 kinds of drugs (cholinesterase inhibitors and NMDA receptor antagonists) have been clinically approved by the FDA for the treatment of AD, which can only delay symptoms and cannot change the progression of the disease. At present, the pathogenesis of $\mathrm{AD}$ has not been clearly clarified. In view of this situation, governments and R \& D institutions of pharmaceutical enterprises attach great importance to the development of AD drugs. Data show that between 1998 and 2017, 146 clinical research and development centers for Alzheimer's disease around the world failed. This article reviews the daily use of AD drugs and the progress of drug research and development, hoping to provide some ideas for medical workers who understand AD drugs and researchers who develop new drugs.
\end{abstract}

Keywords: Alzheimer's disease; Clinical drugs; New drug research and development

\section{Introduction}

Alzheimer's disease (AD) is a degenerative disease of the central nervous system associated with familial heredity and aging. According to the "World Alzheimer Report 2019 Attitudes to dementia" report released by the International Alzheimer's Disease (ADI), the number of $\mathrm{AD}$ patients worldwide exceeded 50 million in 2019, and this number will increase to 152 million by 2050. Since the global cost of treatment for Alzheimer's disease is about \$1 trillion, it will increase to $\$ 2$ trillion by 2030 [1]. According to the Survey of Alzheimer's Disease patients and Family living conditions in China, the number of AD patients in China was 10 million in 2019, which has become the region with the largest number and fastest growth rate of $\mathrm{AD}$ patients in the world [2], while the per capita annual social and economic cost of AD patients in China is as high as \$19144.36 [3]. Governments and R \& D institutions of pharmaceutical companies are working hard for this global public health crisis, but there has been no new drug on the market for 17 years since the US FDA approved Memantine in 2003. On November 2, 2019, the China Food and Drug Administration (CFDA) approved the application of mannitol sodium (code name GV-971), a new drug for the treatment of AD, to be listed in Shanghai Green Valley Pharmaceutical Co., Ltd., changing the history that there are no new drugs on the market in this field. 


\section{Clinical Drug Treatment of Alzheimer's Disease}

\section{Cholinesterase inhibitor (AChEIs)}

Acetylcholinesterase (AChE) is a key hydrolase in the conduction process of the central nervous system. It can quickly hydrolyze acetylcholine into acetic acid and choline in the synaptic space, preventing the transmission of impulses. The increase of acetylcholinesterase in the brain can lead to the decrease of the content of acetylcholine, and the decrease of acetylcholine is one of the main reasons for AD [4]. AChEI can inhibit cholinesterase, reduce the hydrolysis of $\mathrm{ACh}$, increase the level of synaptic $\mathrm{ACh}$ in the brain of patients, and improve the learning and cognitive function of patients with mild to moderate AD. At present, this kind of drug has been widely recognized in clinic [5].

Tacrine: Tacrine belongs to the first generation of nonselective AChEI, the first drug specifically approved by the US Food and Drug Administration (FDA) to treat mild to moderate AD. A large dose of $50-160 \mathrm{mg} / \mathrm{d}$ can delay $25 \%$ to $50 \%$ of patients and improve memory [6]. Due to peripheral cholinergic irritation and hepatotoxicity, the clinical application of this drug is limited [7].

Donepezil: Donepezil belongs to the second generation of selective non-competitive reversible AChEI. In addition, it also has a neuroprotective effect, promoting nerve regeneration in the hippocampus [8], improving patients' cognition and improving patients' activities of daily living [9]. Donepezil has high selectivity to central neurons. After administration, the blood concentration of $3 \mathrm{~h} \sim 5 \mathrm{~h}$ reached the highest, the half-life is longer $(70 \mathrm{~h} \sim 80 \mathrm{~h})$, and the adverse reactions are short, mild and transient. At present, it is recommended to take it before going to bed, the initial dose is $5 \mathrm{mg} \bullet \mathrm{d}-1$, the maximum dose is $10 \mathrm{mg} \bullet \mathrm{d}-1$, for the treatment of mild to moderate AD patients. In 2010, FDA approved high-dose Donepezil tablets (23 mg・d -1) for the treatment of moderate and severe AD patients. In phase III clinical trials, high-dose Donepezil showed good safety and tolerance [10]. At present, drugs are widely used in clinic to treat Alzheimer's disease.

Rivastigmine: Rivastigmine, also known as rivastigmine, is a dual inhibitor of acetylcholinesterase (AChE) and butyrylcholinesterase (BuChE), which can reduce the neurotoxicity of amyloid plaque and has neuroprotective effect [11]. It has a high selectivity to the hippocampus and cerebral cortex, can increase the concentration of acetylcholine in the synaptic space of nerve cells, and improve the cognitive function and mental symptoms and scores of patients [12]. At present, it is recommended that the initial dose of the capsule is $3 \mathrm{mg} / \mathrm{d}$, once in the morning and evening, the maximum tolerance dose should be determined according to the patient's tolerance, each dose should last at least 2 weeks, and the maximum recommended dose is $12 \mathrm{mg} / \mathrm{d}$. The initial dose of transdermal patch is $4.6 \mathrm{mg} / \mathrm{d}$, and the maintenance dose is 4 weeks. For patients with mild to moderate AD, $9.5 \mathrm{mg} / 24 \mathrm{~h}$ is recommended for patients with good tolerance. The high-dose transdermal patch $(13.3 \mathrm{mg} / 24 \mathrm{~h})$ approved by FDA in 2012 can significantly improve the language ability of $\mathrm{AD}$ patients in the treatment of severe AD. Compared with oral administration, the incidence of adverse reactions of transdermal administration was lower and the compliance of patients was higher [13]. At present, there are oral liquid, oral capsule, transdermal patch and other dosage forms. The diversification of dosage forms has led to the continuous expansion of the scope of clinical application of Rivastigmine.

Galantamine: Galantamine belongs to the second generation of reversible AChEI inhibitors and nicotinic receptor regulators, which has dual regulatory effects on the body. The drug has high selectivity to neuronal cholinesterase, can delay the progression of $\mathrm{AD}$ disease, effectively improve cognitive decline [14], antagonize the damage of $\beta$-amyloid protein, and then play a neuroprotective effect [15]. Oral 1 hour after meal, the initial dose is $5 \mathrm{mg}$, four times a day, adjusted to $10 \mathrm{mg}$ after three days, four times a day. The drug can be used in patients with ineffective or poorly tolerated Donepezil [16].

Huperzine A: Huperzine A, a sesquiterpene alkaloid, was extracted from Huperziaceae of Chinese medicine Lycopodii Serrati Herba by Prof. Tang Xican from Shanghai Medical Research Institute of Chinese Academy of Sciences. It is a highly selective, reversible, efficient and low toxic acetylcholinesterase inhibitor, which can pass through the blood-cerebrospinal fluid barrier and reduce acetylcholinesterase activity. Reduce the decomposition of Ach, significantly increase the content of acetylcholine in the brain tissue, and then enhance the learning and memory function of the brain region [17]. $0.1 \mathrm{mg} \sim 0.2 \mathrm{mg}$ once, twice a day, and daily dose should not exceed $0.45 \mathrm{mg}$.

\section{Glutamate receptor antagonist}

Akatinol Memantine N-methyl-D-aspartate (NMDA) receptor antagonist, the first drug approved by FDA for the treatment of moderate to severe AD. Excessive glutamate in patients with $\mathrm{AD}$ leads to excitotoxicity and increases neuronal calcium load, which in turn leads to neuronal damage. Memantine can prevent nerve cell apoptosis, improve patients' memory ability, and stimulate dopamine receptors to improve patients' motor function [18]. A large number of studies have shown that the protective effect of memantine on neurons can be achieved in $\mathrm{AD}$ animal model experiments. The maximum daily dose was $20 \mathrm{mg}$. In order to reduce the occurrence of adverse reactions, the maintenance dose was gradually reached according to the weekly increasing dose 5 mg 3 weeks before treatment. For example, daily $5 \mathrm{mg}$ (1/2 tables, morning) the first week. In the second week, $10 \mathrm{mg}$ per day $(1 / 2$ tables, 2 times a day). Daily $15 \mathrm{mg}$ in the third week (1tablet in the morning, half in the afternoon). Starting from the fourth week, the recommended maintenance dose is $20 \mathrm{mg}$ ( 1 tablet once, twice a day). It can be taken on an empty stomach or with food. In a postmarket clinical study, Meijingang looked at adverse events such 
as dizziness, constipation, high blood pressure and drowsiness. Guidelines issued by the Federation of European Neurological Societies (ENFS) in 2012 indicate that the combination of memantine and AChEIs can be more effective in the treatment of moderate and severe AD [19].

\section{Adjuvant drugs for clinical application}

\section{Free radical scavengers and antioxidants}

Individual cells continue to produce free radicals in the process of metabolism, excessive free radicals damage the body's cells and tissues, damage the central nervous system, leading to aging and chronic diseases [20]. At the same time, some studies have shown that, as a key part of learning and memory, the content of free radicals in the hippocampus is the highest [21]. Melatonin is an endogenous free radical scavenger that protects against nerve injury and improves learning and memory [22]. Oral administration of $3 \mathrm{mg}$ before going to bed every night can regulate the sleep cycle of $\mathrm{AD}$ and reduce the number of nocturnal awakening. Selegiline is a monoamine oxidase inhibitor. It antagonizes oxidative stress related to dopamine catabolism and reduces the production of free radicals. The initial dose is $5 \mathrm{mg}$, which can be increased to $10 \mathrm{mg}$ a day. Ginkgo biloba extract scavenged excessive free radicals, inhibited lipid peroxidation of cell membrane, improved blood circulation, and increased the supply of oxygen and glucose in ischemic tissue, $40 \sim 80 \mathrm{mg}$ each time, 2 3 times a day. Both vitamin $\mathrm{C}$ and vitamin $\mathrm{E}$ are used to alleviate memory and cognitive function in patients with $\mathrm{AD}$.

\section{Improving brain metabolic activator}

These drugs are amide central stimulants acting on the cerebral cortex, which can not only promote the utilization of phospholipids, amino acids and glucose in the brain, but also increase the synthesis of brain proteins and improve various types of brain hypoxia and brain injury, and improve the responsiveness, excitability and learning and memory ability of patients [23]. The representative drugs were Piracetam 0.8-1.6 g, 3 times a day for 1-2 months, Oxiracetam $800 \mathrm{mg}, 2-3$ times a day, and Aniracetam 0.1-0.2 g, 3 times a day for 1-2 months as a course of treatment.

\section{Other}

Irdibenone, an oxidoreductase inhibitor, can activate the respiratory activity of brain mitochondria, improve energy metabolism and glucose utilization after cerebral ischemia, and increase the production of ATP in the brain [24], 30mg each time, 3 times a day, after meals. Nimodipine, a Ca2+ antagonist, can protect and promote memory and intellectual recovery and increase cerebral blood flow, 30-120 mg, 3 times a day. Estrogen can inhibit neuroinflammation, regulate cholinergic system and neuroprotection, and improve memory impairment [25]. Other adjuvant drugs are also used to treat cognitive impairment in patients with Alzheimer's disease.

\section{Research and Development of New Drugs for Alzheimer's Disease}

Governments and major pharmaceutical companies have invested huge amounts of research and trial money in the research and development of drugs to treat AD. The National Institutes of Health's funding provided more than $5 \%$ of the total budget for AD in 2019 [26]. All countries are making great efforts to develop comprehensive, multi-field and multi-channel AD new drugs. In recent years, Pfizer / Johnson's bapineuzumab, Lilly's Solanezumab, Mercer's Verubecestat, Lilly's and AstraZeneca's Lanabecestat all failed in the III phase [27], which made major pharmaceutical companies cautious about AD drug research and development, and even Pfizer announced in February 2018 that it would stop drug research and development work for the disease. Although we have encountered some setbacks, we are still doing our best to move forward. GV-971 (mannitol sodium), jointly developed by Ocean University of China, Shanghai Institute of Medicine of the Chinese Academy of Sciences and Shanghai Green Valley Pharmaceutical Co., Ltd., has become the first drug approved for the treatment of Alzheimer's disease since 2003. In non-clinical trials, the drug suppresses neuroinflammation and reverses cognitive impairment by significantly altering the composition of intestinal microflora and regulating amino acid metabolism, including significant reductions in phenylalanine and isoleucine levels. In clinical trials, AD patients have a good tolerance to low mannose sodium. In the safety analysis (mannosaccharic acid group $n=1577$, placebo group $n=16495$ ), there was no significant difference in overall adverse reactions between the sodium oligoglycerate group and the placebo group [28,29]. The success of the development of GV-971, so that the scientific research team fighting in the front line of $\mathrm{AD}$ drug development saw the dawn.

Aducanumab is a monoclonal antibody targeting $\beta$-amyloid protein and oligomer. In $\mathrm{AD}$ transgenic mice, $A \beta$ decreased in a dose-dependent manner. Further analysis, including the longer exposure of participants to higher antibody doses, showed that Aducanumab monoclonal antibodies reduced brain amyloid protein, nearly half of the patients no longer had positive amyloid PET scans, and plaques were significantly reduced in all examined cortical regions. Based on the above data analysis, Biogen submitted a listing application to FDA [30,31]. Professor Yu Jin of Huashan Hospital affiliated to Fudan University said: "Early intervention and treatment of mild cognitive impairment caused by $\mathrm{AD}$ is hopeful to prevent the onset of AD." The limitations of the understanding of biomarkers make the current drugs or researchers mainly focus on clinically diagnosed diseases. $\mathrm{AD}$ as a progressive central nervous system disease, pathological changes may have occurred before the emergence of clinical syndrome. At the International Conference of the Alzheimer's Association (AAIC-2020), the teams of the United States and Sweden announced that the jurisdiction of testing P-tau 217 in subjects was $89-98 \%$, and that some patients with early- 
onset familial AD had elevated P-tau217 20 years before the onset of symptoms, and blood tests could be diagnosed earlier [32]. Early diagnosis and early treatment can inhibit or even reverse the progression of the disease. The establishment of a new detection method may be one of the successful methods for the development of anti-AD drugs in the future. Other three-phase anti-AD drugs approved by the FDA, such as anti-A $\beta$ drugs BAN2401 [33], CAD106 [34], Gantenerumab [35], new drugs targeting Tau protein TRX0237 [36], neurotransmitter drugs Bresiprazole, Esitopram [37] and so on. According to FDA's website ClinicalTrials.gov, clinical trials of 121 drugs to treat Alzheimer's disease are under way in the nearly five years to Feb. 27, 2020. A total of 29 drugs were identified in 36 phase III clinical trials. Of the 121 experimental drugs, 12 are designed to enhance cognition and 12 are designed to treat neuropsychiatric and behavioral symptoms. Ninety-seven drugs are being tested to improve the disease. $16.5 \%$ contained amyloid protein and $11.3 \%$ took tau as the main target. Compared with 2019, the number of disease modifiers targeting pathways other than amyloid or Tau has increased [37]. All of these have the potential to become international drugs for the treatment of AD. The world is looking forward to the release of detailed clinical trials, hoping to benefit patient $\mathrm{s}$ around the world as soon as possible.

Of course, for diseases caused by a variety of factors, such as $\mathrm{AD}$, clinical trials aimed at a particular cause or targets often end in failure. For example, it is difficult to develop a new drug for a certain pathological characteristic; most of the early animal trials of the drug are effective for phase III clinical failure, and there are limitations in animal models and so on. These failed experiences also provide valuable experience for new drug developers According to the experience of previous failures and the deepening of the pathological mechanism of $\mathrm{AD}$, researchers continue to adjust the ideas of drug research and development, and the research and development of anti-AD drugs is still full of hope.

\section{Conclusion}

$\mathrm{AD}$ is an age-related progressive neurodegenerative disease characterized by early-onset neuronal death. Especially in the past 20 years, research on the pathogenesis of $\mathrm{AD}$ and clinical drug treatment has been developed rapidly. Cholinesterase inhibitors like Donepezil and glutamatergic receptor antagonists like Memantine are the main therapeutic drugs for relieving $\mathrm{AD}$ symptom. Meanwhile, traditional Chinese medicine like Ginkgo biloba extract and $\mathrm{Ca} 2+$ antagonists like Nimodipine are also used to delay the development of AD. However, these clinical strategies for $\mathrm{AD}$ are still lack of ideal achievement. All over the world medical scientists are devote their efforts to seek the safe and effective anti-AD drugs by a comprehensive, multi-field and multi-channel manner. Then, a long pathway need to be forward to perform for new drug research and development, which also requires the governments and major pharmaceutical companies to join the battle for the treatment of AD.

\section{Acknowledgement}

Authors thank Hebei Provincial Natural Science Foundation (No. C2009001007, H2014406048, H2019406063), Hebei Provincial Administration of Traditional Chinese Medicine (No. 05027, 2014062), Hebei Provincial Education Department (No. ZD20131022, ZD2019057), Hebei Provincial Hundred Outstanding Innovated Talents, First Batch and the Key Subject Construction Project of Hebei Provincial College, The key development subject of Pharmacology of Traditional Chinese Medicine of Chengde Medical College, Science and Technology Innovation Team Construction Project of Chengde Medical College of China for financial supports.

\section{Conflict of Interest}

No conflict of interest.

\section{References}

1. Alzheimer's Disease International (2019) World Alzheimer Report 2019: Attitudes to dementia. London.

2. (2020) An investigation report on the family living conditions of patients with Alzheimer's disease in China in 2019. Beijing.

3. Jia J, Wei C, Chen S, Li F, Tang Y, et al. (2018) He cost of Alzheimer's disease in China and re-estimation of costs worldwide. Alzheimer's \& Dementia 14(4): 483-491.

4. Akıncıoğlu H, Gülçin İ (2020) Potent acetylcholinesterase Inhibitors: Potential drugs for Alzheimer's disease. Mini Rev Med Chem 20(8): $703-$ 715 .

5. Huang M, Wang QW, Chen HM (2014) Study on the treatment of cholinesterase inhibitors in Alzheimer's disease. Modern Practical Medicine 26(6): 779-781.

6. Knopman D, Schneider L, Davis K, F Smith, T Hoover, et al. (1996) Longterm tacrine (Cognex) treatment: effects on nursing home placement and mortality, tacrine study group. Neurology 47(1): 166-177.

7. Chen XM, Wang YH (2001) New progress in the treatment of Alzheimer's disease. Chinese General Practice (12): 940-942.

8. Man J, Cui K, Fu X, Zhang D, Lu Z, et al. (2020) Donepezil promotes neurogenesis via Src signaling pathway in a rat model of chronic cerebral hypoperfusion. Brain Research 1736: 146782.

9. Chen K (2016) Clinical trial of donepezil hydrochloride in the treatment of Alzheimer's disease. The Chinese Journal of Clinical Pharmacology 32(11): 981-983.

10. Tariot P N, Salloway S, Yardley J, Joan Mackell, Margaret Moline, et al. (2012) Long-term safety and tolerability of donepezil $23 \mathrm{mg}$ in patients with moderate to severe Alzheimer's disease. BMC Research Notes, 5(1): 283-288.

11. Matsuda T, Hisatsune T (2017) Cholinergic modification of neurogenesis and gliosis improves the memory of A $\beta$ PPswe/PSEN1dE9 Alzheimer's disease model mice fed a high-fat diet. J Alzheimer Dis 56(1): 1-23.

12. Matsuzono K, Sato K, Kono S, Nozomi Hishikawa, Yasuyuki Ohta, et al. (2015) Clinical Benefits of Rivastigmine in the RealWorld Dementia Clinics of the Okayama Rivastigmine Study (ORS). J Alzheimers Dis 48(3): 757-763.

13. Lee JH, Sevigny J (2011) Effects of body weight on tolerability of rivastigmine transdermal patch: a post-hoc analysis of a double-blind trial in patients with Alzheimer disease. Alzheimer Dis Assoc Disord 25(1): 58-62.

14. Kavanagh S, Van Baelen B, Schauble B, et al. (2011) Long-term effects of galantamine on cognitive function in Alzheimer's disease: a large-scale international retrospective study. J Alzheimers Dis 27(3): 521-530. 
15. Li QQ Yang SS, Huang S (2017) Protective effects and mechanisms of galantamine on neurotoxicity induced by $\beta$-Amyloid protein. Journal of Brain and Nervous Diseases 25(2): 110-115.

16. Engedal K, Davis B, Richarz U, J Han, B Schäuble, et al. (2012) Two galantamine titration regimens in patients switched from donepezil. Acta Neurol Scand 126(1): 37-44.

17. Damar U, Gersner R, Johnstone JT, S Schachter, A Rotenberg, et al. (2016) Huperzine $\mathrm{A}$ as a neuroprotective and antiepileptic drug: a review of preclinical research. Expert Rev Neuroth 16(6): 671-680.

18. Zhang J, Cao YF, Li WL (2016) Analysis of the efficacy of acetylcholinase inhibitors combined with memang hydrochloride in the treatment of senile dementia. Journal of Clinical Medicine in Practice 20(21): 136138.

19. Sorbi S, Hort J, Erkinjuntti T, T Fladby, G Gainotti, et al. (2012) EFNS-ENS Guidelines on the diagnosis and management of disorders associated with dementia. Eur J Neurol 19(9): 1159-1179.

20. Wang XZ, Wang R (2019) The pharmacotherapy and research progress of drugs for Alzheimer's disease. Chinese Journal of New Drugs 28(24): 2960-2964.

21. Huang XD, Moir RD, Tanzi RE, Ashley I Bush, Jack T Rogers (2004) Redoxactive metals, oxidative stress, and Alzheimer's disease pathology. Ann N Y Acad Sci 1012: 153-163.

22. Yang L (2017) Role and mechanism of melatonin and its receptor MT2 in learning and memor y. Fourth military Medical University.

23. Wang J, Zhang J, Zhou J (2016) Systematic review on curative effects of oxiracetam and piracetam in treatment of cerebral organic syndrome. Chinese Journal of Evidence-Based Cardiovascular Medicine 8(2): 160163.

24. Yang H, Yi XP, Ji ZH (2019) Progress in the application of idebenone in the treatment of nervous system diseases. Electronic Journal of Clinical Medical Literature 6(4): 188-190.

25. Chakrabarti M, Haque A, Banik NL, Prakash Nagarkatti, Mitzi Nagarkatti, et al. (2014) Estrogen receptor agonists for attenuation of neuroinflammation and neurodegeneration. Brain Res Bull 109: 22-31.
26. Hu DL (2018) Bet on Alzheimer's disease. World Science (12): 48-51.

27. Lin TT, Zhan YX, Fu SM, et al. (2018) The progress of drug discoveries for Alzheimer's disease. Journal of University of Science and Technology of China 48(10): 825-837.

28. Syed YY. Sodium 0 ligomannate: First approval. Drugs 80(4): 441-444.

29. Wang XY, Sun GQ, Feng T, et al. (2019) Sodium oligomannate therapeutically remodels gut microbiota and suppresses gut bacterial amino acids-shaped neuroinflammation to inhibit Alzheimer's disease progression. Cell Res 29(10): 787-803.

30. Sevigny J, Chiao P, Bussière T (2016) The antibody aducanumab reduces A $\beta$ plaques in Alzheimer's disease. Nature 537(7168): 50-56.

31. Schneider L (2020) A resurrection of aducanumab for Alzheimer's disease. Lancet Neurol 19(2): 111-112.

32. Palmqvist S, Janelidze S, Quiroz YT, Zetterberg T, Francisco Lopera, et al. (2020) Discriminative accuracy of plasma phospho-tau 217 for Alzheimer disease vs other neurodegenerative disorders. JAMA 28: E1E10.

33. Abbasi J (2018) Promising results in 18-month analysis of Alzheimer drug candidate. JAMA 320(2): 965

34. Hameed S, Fuh JL, Senanarong V, Esther Gunaseli M. Ebenezer, Irene Looi, et al. (2020) Role of fluid biomarkers and PET imaging in early diagnosis and its clinical implication in the management of Alzheimer's disease. J Alzheimers Dis Rep 4(1): 21-37.

35. Ostrowitzki S, Lasser RA, Dorflinger E, Philip Scheltens, Frederik Barkho et al. (2017) A phase III randomized trial of gantenerumab in prodromal Alzheimer's disease. Alzheimers Res Ther 9: 95.

36. Lee HE, Lim D, Lee JY, Sang M Lim, Ae N Pae (2019) Recent tau-targeted clinical strategies for the treatment of Alzheimer's disease. Future Med Chem 11(15): 1845-1848.

37. Cummings J, Lee G, Ritter A (2020) Alzheimer's disease drug development pipeline: 2020. Alzheimers Dement (N Y) 6(1): e12050. 